\title{
Global dissemination of Influenza A virus is driven by wild bird migration through arctic and subarctic zones
}

\author{
Jonathon Gass ${ }^{1}$
}

${ }^{1}$ Affiliation not available

February 1, 2022

Authors: Jonathon D. Gass Jr. ${ }^{1,+}$, Robert J. Dusek ${ }^{2}$, Jeffrey S. Hall ${ }^{2}$, Gunnar Thor Hallgrimsson ${ }^{3}$, Halldór Pálmar Halldórsson ${ }^{4}$, Solvi Runar Vignisson ${ }^{4,5}$, Sunna Bjork Ragnarsdottir ${ }^{6,7}$, Jón Einar Jónsson ${ }^{8}$,

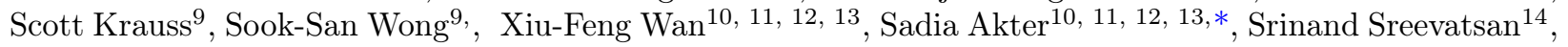
Nídia S. Trovão ${ }^{15}$, Felicia B. Nutter ${ }^{1}$, Jonathan A. Runstadler ${ }^{1}$, Nichola J. Hill ${ }^{1,16}$

${ }^{1}$ Department of Infectious Disease and Global Health, Cummings School of Veterinary Medicine, Tufts University

${ }^{2}$ U.S. Geological Survey, National Wildlife Health Center

3 Institute of Biology, University of Iceland

${ }^{4}$ University of Iceland's Research Centre in Suurnes

${ }^{5}$ Suurnes Science and Learning Center

${ }^{6}$ Icelandic Institute of Natural History

7 The Southwest Iceland Nature Research Centre

${ }^{8}$ University of Iceland's Research Center at Snæfellsnes

9 Department of Infectious Diseases, St. Jude Children's Research Hospital

${ }^{10}$ Center for Influenza and Emerging Infectious Diseases (CIEID), University of Missouri, Columbia

${ }^{11}$ Department of Molecular Microbiology and Immunology, University of Missouri, Columbia

12 Bond Life Sciences Center, University of Missouri, Columbia

13 Department of Electronic Engineering and Computer Science, University of Missouri, Columbia

14 College of Veterinary Medicine, Michigan State University

15 Division of International Epidemiology and Population Studies, Fogarty International Center, National Institutes of Health

${ }^{16}$ Department of Biology, University of Massachusetts, Boston

+ Corresponding author

Corresponding author email: Jonathon.gass@tufts.edu

Present address: State-Key Laboratory of Respiratory Diseases, Guangzhou Medical University and School of Public Health, Li Ka Shing Faculty of Medicine, The University of Hong Kong * Present address: Department of Molecular Microbiology, Washington University School of Medicine in St. Louis 


\begin{abstract}
Influenza A viruses (IAV) circulate endemically among many wild aquatic bird populations that seasonally migrate between wintering grounds in southern latitudes to breeding ranges along the perimeter of the circumpolar arctic. Arctic and subarctic zones are hypothesized to serve as ecologic drivers of the intercontinental movement and reassortment of IAVs due to high densities of disparate populations of long distance migratory and native bird species present during breeding seasons. Iceland is a staging ground that connects the East Atlantic and North Atlantic American flyways, providing a unique study system for characterizing viral flow between eastern and western hemispheres. Using Bayesian phylodynamic analyses, we sought to evaluate the viral connectivity of Iceland to proximal regions and how inter-species transmission and reassortment dynamics in this region influence the geographic spread of low and highly pathogenic IAVs. Findings demonstrate that IAV movement in the arctic and subarctic reflects wild bird migration around the perimeter of the circumpolar north, favoring short-distance flights between proximal regions rather than long distance flights over the polar interior. Iceland connects virus movement between mainland Europe and North America, consistent with the westward migration of wild birds from mainland Europe to Northeastern Canada and Greenland. Though virus diffusion rates were similar among avian taxonomic groups in Iceland, gulls play an outsized role as sinks of IAVs from other avian hosts prior to onward migration. These data identify patterns of virus movement in northern latitudes and inform future surveillance strategies related to seasonal and emergent IAVs with potential public health concern.
\end{abstract}

Keywords: Influenza A virus, Iceland, wild birds, phylodynamics, transmission, subarctic

\title{
Introduction
}

Understanding the ecologic interactions among wild birds that facilitate the maintenance, reassortment, and dispersal of influenza A viruses (IAV) is key to forecasting global spread (Hall et al., 2014; Hill et al., 2017; Hoye et al., 2011; Lang et al., 2016; The Global Consortium for H5N8 and Related Influenza Viruses, 2016). IAVs circulate endemically among wild aquatic migratory bird populations, including Anseriformes (waterfowl, including ducks, geese, and swans) and Charadriiformes (seabirds, including gulls, shorebirds, terns, and auks), many of which migrate between breeding ranges in arctic and subarctic regions (ASRs) to wintering grounds in southern latitudes (Dusek et al., 2014; G. A. Gudmundsson, 1993; L. A. Gudmundsson, 1992; Hall et al., 2014; Hall et al., 2015; Hall et al., 2013; Hénaux, Parmley, Soos, \& Samuel, 2013; Lang et al., 2016; Runstadler, Hill, Hussein, Puryear, \& Keogh, 2013; van Dijk et al., 2014). During migration, birds use staging and breeding grounds within the circumpolar perimeter of the arctic circle, where populations from different geographic origins aggregate at high densities, enabling direct and environmental fecal-oral transmission of IAVs within and between species (Causey \& Edwards, 2008; R. Chen \& Holmes, 2009; Hill et al., 2016; Ito et al., 1995). The mass migration of birds to ASRs each spring is considered an important mechanism for inter-species transmission and global spread of IAVs, although empirical data are often lacking from these remote, high latitude regions where human populations are sparse (Causey \& Edwards, 2008; Hoye, Munster, Nishiura, Klaassen, \& Fouchier, 2010; Olsen et al., 2006).

Previous studies support that migratory connectivity throughout ASRs dictate the ecology and evolution of IAVs between hemispheres (Webster, Marra, Haig, Bensch, \& Holmes, 2002). Northern latitudes are predicted to have a high risk of IAV outbreaks among wild birds due to the increased density of disparate bird populations during spring migration and the persistence of IAVs in low temperature climate regimes (Herrick, Huettmann, \& Lindgren, 2013; A. M. Ramey et al., 2020; Runstadler et al., 2013). Host movement patterns within ASRs also determine how viruses disperse within the arctic circumpolar region and onward transport to southern latitudes globally. During post-breeding migration, wild birds such as shorebirds, gulls, skuas, and terns prioritize short distance flights around the perimeter of the greater polar arctic circle to avoid navigation challenges (Alerstam et al., 2007), exemplifying the ecologic importance of stopover and staging locations for migratory seabirds within ASRs. This great-circle orientation of seabird migration through ASRs is likely to have important but currently under-studied impacts on the directionality and rate of movement of IAVs in space and time across the globe. ASRs are also disproportionately affected by global climate change, where observed and predicted annual mean warming is more than twice the global 
mean (Arctic Monitoring and Assessment Programme (AMAP), 2019). Alterations to climate regimes may impact the distribution of wild birds and IAVs throughout the circumpolar north due to geographic shifts in habitat use and trophic food webs, changes in timing of migrations and breeding range locations, and declines in reproductive success (Mckinney et al., 2015). Increased surveillance in northern latitudes will enable researchers to gather baseline data with which to measure future shifts in IAV dynamics because of the rapidly changing arctic climate (Gilbert, Slingenbergh, \& Xiao, 2008; Jensen et al., 2008).

The migratory connectivity of ASRs with the rest of the globe is particularly relevant given the expanding geographic range of highly pathogenic avian influenza (HPAI) virus subtype $\mathrm{H} 5 \mathrm{Nx}$ of clade 2.3.4.4 which was first detected outside of China in 2014 (Antigua, Choi, Baek, \& Song, 2019; Lee, Bertran, Kwon, \& Swayne, 2017). HPAI clade 2.3.4.4 viruses have evolved by reassorting with low pathogenic viruses throughout Eurasia and North America, and evidence of intercontinental dissemination events through ASRs have been documented (Hill et al., 2017; Lee et al., 2017). In 2014, HPAI H5N8 of clade 2.3.4.4 was introduced by wild migratory birds from East Asia to North America via the trans-Beringian route through Alaska, from which H5N2 and H5N1 reassortant viruses emerged (Hill et al., 2017; Lee et al., 2015; A. Ramey et al., 2016). H5N2 reassortant viruses ultimately spread among domestic poultry throughout 15 states in the US, which precipitated the culling of approximately 49 million chickens and turkeys (Hill et al., 2017; Lee et al., 2016). Future introduction events are anticipated in view of the large number of clade 2.3.4.4 outbreaks in areas adjacent to North America, although the exact route of introduction remains elusive (Ramey et al., 2021).

Transcontinental virus exchange is often detected in oceanic and coastal regions at continental margins along migratory flyways (Alerstam et al., 2007; Huang et al., 2014; Pearce et al., 2009). In the Pacific Rim, Alaska has emerged as an ecologically important 'hot spot' through which IAV carrier species migrate, connecting East Asia and North America for virus exchange (Hill et al., 2017; Huang et al., 2014; Lee et al., 2015; A. Ramey et al., 2015; A. Ramey et al., 2016). Though significant westward virus migration from Asia to Europe has been documented, far less is known about the onward viral connectivity of mainland Europe with staging locations within the North Atlantic and North America (Kilpatrick et al., 2006; Olsen et al., 2006). Addressing how IAV dynamics may differ between the North Atlantic and Pacific coastal regions relative to global patterns of viral flow is critical for developing a well-rounded understanding of influenza ecology. The current knowledge gap highlights the potential for undetected virus spread between Europe and North America, especially for HPAI clade 2.3.4.4 and other highly consequential influenza viruses.

Iceland - at the confluence of the North Atlantic and Arctic oceans - provides a unique study system for characterizing inter-hemispheric viral flow between eastern and western hemispheres. The island is situated within the East Atlantic flyway between Europe and North America where resident and long-distance migratory birds interact (Butler, 2012; Dusek et al., 2014; Hall et al., 2014; Hall et al., 2015; Newton, 2008). Viruses of North American and Eurasian ancestral lineages, as well as reassortant strains between these lineages, have been isolated in Iceland, demonstrating the North Atlantic as a potential corridor for the inter-hemispheric movement of IAVs (Dusek et al., 2014). Previous research has described (a) high seroprevalence of IAVs among North Atlantic sea ducks (Hall et al., 2015) and (b) genes from HPAI virus lineages in low pathogenic IAVs among migrant birds in Iceland, revealing the importance of surveillance in the North Atlantic for viruses with pandemic potential in humans (Hall et al., 2014). There is very little evidence, however, describing the directionality of virus movement and avian host transmission dynamics within Iceland and throughout the North Atlantic region, which may influence the spread of IAV globally (Dusek et al., 2014; Hall et al., 2014; Hall et al., 2015; Kilpatrick et al., 2006; The Global Consortium for H5N8 and Related Influenza Viruses, 2016).

We hypothesize that IAVs are transported via wild bird migration between Europe and North America through Iceland and that Iceland plays an important role in the inter-hemispheric transmission and reassortment of IAVs in the North Atlantic, particularly with virus moving into North America. This study examines (i) viral connectivity of Iceland to other global regions, (ii) inter-species transmission dynamics among wild aquatic birds in this system, (iii) viral diffusion by wild aquatic birds within Iceland compared to other global regions, and (iv) whether local reassortment patterns may result in novel or HPAI reassortants with 
the potential for inter-hemispheric transport, warranting public health concern. These data reveal patterns of inter-hemispheric virus movement and inform surveillance efforts related to seasonal and emergent IAVs, in northern latitudes, particularly throughout the North Atlantic region.

\section{Materials and Methods Field sample collection}

From May 2010 through February 2018, we obtained IAV isolates from various species of seabirds, shorebirds, and waterfowl as well as environmental sampling of avian fecal material from locations throughout Iceland (Dusek et al., 2022). Live sampled birds were captured using a $18 \mathrm{~m} \times 12 \mathrm{~m}$ cannon-propelled capture net, noose pole, or hand capture. Birds found dead or moribund were also sampled. Hunter-harvested waterfowl and fisheries-bycatch seabirds were sampled as available. All birds were identified to species and, for live birds, the majority were individually marked with metal bands. Age characteristics were determined and age was documented for each bird according to the following schemes adapted from U.S. Geological Survey (USGS) year classification codes: hatched in same calendar year as sampling $(1 \mathrm{CY})$, hatched previous calendar year $(2 \mathrm{CY})$, hatched previous calendar year or older exact age unknown $(2 \mathrm{CY}+$; i.e. at least $2 \mathrm{CY}$ but could be any age older then $2 \mathrm{CY}$ as well), hatched three calendar years prior to sampling (3CY), hatched four calendar years prior to sampling $(4 \mathrm{CY})$, hatched more than four calendar years prior to sampling $(4 \mathrm{CY}+)$, or unknown if age could not be determined (U) (Olsen KM, 2004; Prater, Marchant, \& Vuorinen, 1977; USGS, 2020). Due to species specific differences, not all aging categories could be applied to all species sampled. All live birds were immediately released following completion of sampling.

To sample for IAV, a single polyester-tipped swab was used to swab the cloaca only (2010-2013) or to first swab the oral cavity then the cloaca (2014-2017). Opportunistic environmental sampling of fecal material was also conducted using a direct swabbing method (2018). Each swab sample was immediately placed in individual cryovials containing $1.25 \mathrm{ml}$ viral transport media (Docherty \& Slota, 1988). Vials were held on ice for up to 5 hours prior to being stored in liquid nitrogen or liquid nitrogen vapor. Samples were shipped on dry ice from Iceland to Madison, Wisconsin, USA by private courier with dry ice replenishment during shipping. Once received in the laboratory, samples were stored at $-80^{\circ} \mathrm{C}$ until analysis.

Virus extraction, RT-PCR, virus isolation

Viral RNA was extracted from swab samples using the MagMAX ${ }^{\mathrm{TM}}-96$ AI/ND Viral RNA Isolation Kit (Ambion, Austin, TX) following the manufacturer's procedures. Real-time RT-PCR was performed using previously published procedures, primers, and probes (Spackman et al., 2002) designed to detect the IAV matrix gene. RT-PCR assays utilized reagents provided in the Qiagen OneStep ${ }^{\circledR}$ RT-PCR kit (Qiagen, Germantown, MD). Following RT-PCR screening, all samples with $\mathrm{Ct}$ values $<45$ were attempted to be grown in embryonated chicken egg cultures (Woolcock, 2008), the harvested allantoic fluid were tested again by RT-PCR and samples with Ct values $<22$ were selected for sequencing. This cut-off was selected based on our previous experiences, as samples with higher Ct-values typically yield poor quality sequence results. Egg-grown virus isolates were sequenced using multiple standard methods including Sanger (Sanger, Nicklen, \& Coulson, 1977), Roche 454 (Margulies et al., 2005), and Illumina HiSeq 2000 and MiSeq (Illumina, San Diego, CA) sequencing (Dusek et al., 2014; Guan et al., 2019; Hall et al., 2014).

\section{Datasets for phylodynamic analyses}

Global dataset: The polymerase basic protein 2 (PB2) segment was selected as the basis for phylodynamic analysis, given (a) it is the largest internal segment of the IAV genome (maximizing the number of nucleotides in the analysis (>2000 nts)), (b) it is relatively fast evolving for an internal gene with a mean of $3.15 \times 10^{-3}$ (95\% HPD 1.94-4.44) substitutions per site per year (Rubing Chen \& Holmes, 2006), and (c) enables the investigation of transmission dynamics without targeting a specific subtype. The temporal signal of the PB2 gene and clock-like evolution of both global and local datasets were evaluated (Supplementary figure 1). All globally available avian and marine mammal IAV PB2 genes sequenced between 2009 and 2019, excluding those from Iceland, were downloaded from the National Center for Biotechnology Information Influenza Virus Resource database (NCBI IVR) (Bao et al., 2008) on February 12, 2020, resulting in 13,434 sequences. Iceland-derived sequences were downsampled separately, as described below. Duplicate sequences (based 
on collection date, location, and nucleotide content) and sequences with less than $75 \%$ unambiguous bases were removed, and all vaccine derivative and laboratory-synthesized recombinant sequences were excluded. Sequences in the dataset were only included if isolation dates, location, and host species were available, resulting in 7,210 remaining sequences. The subsequent downsampling strategy aimed to reduce the number of sequence taxa for computational efficiency and to mitigate sampling bias while maintaining genetic diversity in the dataset.

Downsampling of global taxa outside of Iceland (i.e. the 'outgroup'): Four variables were considered important for explaining genetic diversity in the global outgroup IAV sequence dataset: geographic region, host taxa, sampling year, and hemagglutinin (HA) subtype. Five geographic region categories included North America, Europe, Asia, Africa, and South America (Australia and Antarctica were removed due to insufficient sequence counts). Fourteen HA subtype categories included H1, H2, H3, H4, H6, H8, H10, H11, H12, H13, H14, H15, $\mathrm{H} 16$, and pooled H5/7/9. H5, H7, and $\mathrm{H} 9$ were combined to reduce bias, as these were over-represented in the global dataset. Four host categories included Anseriformes, Charadriiformes, Galliformes, and Other, which comprised all other avian taxa and marine mammals.

To inform the downsampling strategy for the outgroup and evaluate if any of the four variables were correlated, a multiple correspondence analysis (MCA) was performed (JMP Pro v.14.0.0 (JMP Version 14.0.0, 1989-2019)). The MCA uses categorical data as input, which for this study included the sampling metadata associated with each sequence (region, host taxa, year, and HA subtype). Through representation of the variables in two-dimensional Euclidean space, significant clustering of HA subtypes with host taxa was detected (Supplementary figure 2), indicative of host-specific subtypes that are a well-known feature of influenza (Olson et al., 2014). These findings confirmed by previously published data on species-specificity of HA subtypes (Byrd-Leotis, Cummings, \& Steinhauer, 2017; Long, Mistry, Haslam, \& Barclay, 2019; Verhagen et al., 2015) led us to downsample the dataset stratifying taxa by two non-overlapping variables: geographic region and HA subtype. Downsampling of the global data resulted in 21-75 taxa per five geographic region categories and 6-30 taxa per $14 \mathrm{HA}$ subtype categories, resulting in a total of 301 sequences in the global outgroup, with relative evenness across sampling years. This step was performed to mitigate sampling bias resulting in over-representation of species or viral strains, while accounting for genetic diversity in the dataset.

Downsampling of Iceland-derived taxa (i.e. the 'ingroup'): Next, virus sequences from Iceland (including 35 downloaded from NCBI IVR and 58 novel viruses isolated and recently sequenced by our group $(\mathrm{n}=93)$ ) (Dusek et al., 2022) were downsampled by stratifying taxa by HA subtype (generating 1-15 sequences per 14 HA subtype categories), resulting in 63 sequences. These 63 sequences were used for global and local discrete trait analyses and the ingroup dataset reflected the underlying composition of host-specific subtypes present in this localized system. To assist with rooting and time-calibration of the tree, historical avian sequences from NCBI IVR were downloaded for the years 1979-2008. These were downsampled by year to ensure one sequence per year, resulting in 30 historic sequences. The total downsampled dataset, including the outgroup $(\mathrm{n}=301)$, ingroup $(\mathrm{n}=63)$, and historic sequences $(\mathrm{n}=30)$ resulted in a total of 394 sequences (Gass J.D., 2021).

Europe-Iceland-North America Datasets: Following analyses which identified the most significant geographic regions acting as sources of IAVs to Iceland, a second dataset was constructed at a restricted scale to Europe, Iceland, and North America between 2009 and 2019. First, the cleaned global dataset described above $(\mathrm{n}=7245)$ was downsampled to include significant source regions of North America $(\mathrm{n}=3222)$ and Europe $(\mathrm{n}=407)$, totaling 3629 sequences. To identify at lower spatial resolution the source/sink locations relevant to Iceland, a K-means cluster analysis was performed (JMP Pro v.14.0.0 (JMP Version 14.0.0, 1989-2019)) using latitude/longitude coordinates for each of the 3629 sequences (obtained by extracting sampling location from the strain name of each sequence and searching in www.geonames.org). A total of 20 intraregional clusters resulted. Identified clusters with $<50$ sequences were combined with geographically proximal clusters to increase evenness of within cluster sequence counts for discrete traits analyses, resulting in 13 intraregional cluster locations within North America, Iceland, and the rest of Europe (Supplementary figure 3). 
Viral sequences were then downsampled from 3629 to 743 taxa stratifying by intraregional cluster groupings and HA subtype. Two datasets were formed, both of which included the 743 downsampled sequences (557 from North America, 229 from Europe excluding Iceland), 30 historic sequences (same as the global analysis), and: (i) for discrete trait phylogeographic and phylodynamic analyses (non-geographic analyses of host transmission and subtype reassortment patterns using discrete diffusion models): 63 downsampled Icelandderived sequences, totaling 836 sequences, and (ii) for continuous trait phylogeographic analysis, all 93 Iceland-derived sequences were included, totaling 866 sequences (Gass J.D., 2021). For purposes of clarity, we use the term 'phylogeographic' to refer to analyses that are geographic in nature and 'phylodynamic' to refer to analyses that focus on viral diffusion between non-geographic traits, namely transmission between species and reassortment of HA subtypes. The two datasets were formed because (a) heterogeneity in sampling among locations and host taxa can bias results of discrete trait analyses, therefore downsampling to ensure relative homeogeneity of trait groupings (while striving to preserve host and pathogen population diversity across space and time) is required, and (b) continuous trait analyses are robust against heterogeneity in sampling (Baele, Suchard, Rambaut, \& Lemey, 2017; Lemey, Rambaut, Welch, \& Suchard, 2010), thus the full Iceland dataset was included for continuous analyses (Gass J.D., 2021). Multiple sequence alignments of PB2 sequences were performed using MUSCLE in Geneious Prime 2020.01.02 (https://www.geneious.com) and trimmed to the open reading frame. Maximum-likelihood phylogenies of PB2 segments in the downsampled datasets were reconstructed using RAxML v8.2.12 (Stamatakis, 2006) and temporal signal was investigated using TempEst v1.5.3 (Rambaut, Lam, Max Carvalho, \& Pybus, 2016) (Supplementary figure 1).

\section{Time-scaled Bayesian phylogenetic analyses}

To construct time-scaled trees, Bayesian molecular clock analyses were conducted using the Markov chain Monte Carlo (MCMC) method in BEAST v.1.10.4 (Suchard et al., 2018). Phylogenies were reconstructed using a) a Generalized Time-Reversible model (GTR) of nucleotide substitution (Tavaré \& Miura, 1986) with a gamma distribution of site heterogeneity (also known as the Yang96 model (Yang, 1996)), b) a coalescent-based Gaussian Markov random fields (GMRF) Skyride model for estimating effective population size dynamics (Minin, Bloomquist, \& Suchard, 2008), and c) an uncorrelated relaxed lognormal clock (Drummond, Ho, Phillips, \& Rambaut, 2006). The BEAGLE library was used to optimize computational efficiency (Baele, Ayres, Rambaut, Suchard, \& Lemey, 2019). Eight independent MCMC analyses were run for 200 million generations, sampled every 20,000 runs, and parameter convergence and effective sample size (ESS) (required to be $>200$ ) were evaluated in Tracer v.1.7.1 (Rambaut, Drummond, Xie, Baele, \& Suchard, 2018). Using LogCombiner v.1.10.4, at least $10 \%$ burn-in was removed from each run and independent runs were combined to establish the maximum clade credibility (MCC) tree, from which the last 500 trees from the posterior distribution were extracted and used as the empirical tree for all subsequent phylodynamic analyses (Pagel, Meade, \& Barker, 2004). Trees were visualized using Figtree v1.4.4 (Rambaut A., 2018).

\section{Phylogeographic and phylodynamic analyses}

To infer significant transition rates between epidemiologic features (known as 'traits') of PB2 sequences along phylogenetic tree branches, including (a) geographic location, (b) host species, and (c) HA and Neuraminidase (NA) subtypes, and evaluate continuous virus diffusion (rate of movement in $\mathrm{km} /$ year) among host taxa, joint discrete and continuous trait Bayesian phylogeographic and phylodynamic analyses were performed using BEAST v.1.10.4 (Suchard et al., 2018). An asymmetric substitution model with Bayesian stochastic search variable selection (BSSVS) and a strict clock model were used to estimate diffusion between discrete states (Lemey, Rambaut, Drummond, \& Suchard, 2009). Statistical significance for diffusion between discrete states was determined by the following Bayes Factors (BF): 3 [?] $\mathrm{BF}<20,20$ [?] $\mathrm{BF}<150$, and $\mathrm{BF}$ [?] 150, denoting positive, strong, and very strong support, respectively (Kass \& Raftery, 1995). To quantify transitions among geographic, host, or HA/NA subtype states (Markov jumps, i.e. the frequency of transitions from one geographic or host state to another along phylogenetic branches) and the duration of time viruses spend in each state (Markov rewards), posterior inference of the complete Markov jump history through time was included (Hong, Lemey, Suchard, \& Baele, 2021).

Continuous Bayesian phylogeographic analyses were performed by annotating the empirical tree with latitude 
and longitude locations of all sampling sites via www.geonames.org to infer and visualize the evolutionary and geographic relationships between global sequences. Sequences between 1978 and 2008 were masked so that they contributed to the overall tree structure but were not included in the calculation of diffusion rates globally or within Iceland (Hicks, Dimitrov, Afonso, Ramey, \& Bahl, 2019). A Cauchy relaxed random walk (RRW) model and an uncorrelated relaxed lognormal clock were used and a random jitter of 0.001 was applied to isolates with matching geographic latitude and longitude coordinates (Lemey et al., 2010). Hostspecific diffusion rates, the rate of virus movement (in kilometers per year), were calculated (Dellicour, Rose, \& Pybus, 2016; Minin \& Suchard, 2008; Trovao, Suchard, Baele, Gilbert, \& Lemey, 2015). GIS mapping was performed using QGIS v.3.16.3 (QGIS Development Team, 2020).

\section{Ethical approvals}

This research was conducted under approval of the U.S. Geological Survey National Wildlife Health Center's Animal Care and Use Committee, protocol numbers EP090325, EP120302, and EP153020 in strict accordance with guidelines set forth in the U.S. Government's Animal Welfare Act and the National Institutes of Health Office of Laboratory Animal Welfare. Permits for the capture and sampling of wild birds were issued by the Icelandic Institute of Natural History (Permit Numbers 368 and 403). Permits to ship collected cloacal swab samples from Iceland to the United States (U.S.) were obtained from the Icelandic Institute of Natural History, the U.S. Department of Agriculture, and the US Fish and Wildlife Service. All sampling occurred in conjunction with the Southwest Iceland Nature Research Institute, the University of Iceland, Snaefellsnes Research Centre, the Icelandic Institute of Natural History, and in the case of private lands, with the specific permission of landowners.

\section{Results}

\section{Characterization of Iceland IAV isolates}

Throughout 2010-2018, 6635 waterfowl, seabirds, and shorebirds were sampled (Anseriformes ( $\mathrm{n}=1216)$, Charadriiformes $(\mathrm{n}=5289)$, Galliformes $(\mathrm{n}=75)$, Passeriformes $(\mathrm{n}=4)$, Pelicaniformes $(\mathrm{n}=39)$, and Procellariiformes $(\mathrm{n}=12)$ ) in 34 unique locations throughout Iceland, in the following regions: Southwest $(\mathrm{n}=4747)$, South $(\mathrm{n}=664)$, West $(\mathrm{n}=312)$, East $(\mathrm{n}=457)$, Northeast $(\mathrm{n}=450)$, and Northwest $(\mathrm{n}=5)$. Of the total collected, 381 (5.7\%) tested positive for IAV by RT-PCR, and 94 IAVs were ultimately isolated and sequenced. Thirty-five of the 94 IAV sequences have been previously described (Dusek et al., 2014; Guan et al., 2019; Hall et al., 2014). The PB2 gene of one isolated IAV failed to be sequenced, therefore $93 \mathrm{IAV}$ sequences were included in this analysis. IAVs isolated in Iceland during the sampling timeframe, included 20 distinct subtypes from 92 individual birds (Supplementary table 1a). H6N8, H16N3, H2N5, and H2N7 subtypes were dominant in the dataset, while others were less frequent. The 92 individual birds included 9 species of the order Charadriiformes, and three species of the order Anseriformes, and ranged in age from 1CY to 4CY + (Supplementary Table 1b). Sampling was conducted throughout multiple seasons across years, resulting in sequenced viruses from autumn $(n=72)$, spring $(n=19)$, and winter $(n=1)$ months.

\section{Assessing viral connectivity of Iceland at the global scale}

Global phylogenetic reconstruction of the PB2 segment demonstrated that viruses sampled in Iceland cluster within both the Eurasian and North American clades (Figure 1) and indicated multiple, independent introduction events rather than sustained endemic circulation.

The most significant geographic state transitions from global regions to Iceland occurred via mainland Europe and to a lesser degree North America (Figure 2a), with 18\% of global Markov jumps between mainland Europe and Iceland (which are represented as the percent of global transitions between regional states along phylogenetic tree branches) and to a lesser degree between North America and Iceland (5\% of global Markov jumps). Consistent with the stronger connectivity to Europe relative to North America, IAVs introduced from mainland Europe to Iceland occurred at a rate 3.6 times greater Markov jumps (which are represented as the percent of global transitions between regional states along phylogenetic tree branches) than from North America to Iceland (Figure 2b). Iceland primarily acted as a sink for viruses from other 
high latitude regions, rather than a source. Other global regions were significantly phylogeographically connected, highlighting ecologic and epidemiologic linkages from North America to South America $(\mathrm{BF}=$ $47475)$, Asia to Africa $(\mathrm{BF}=1893.96)$, Asia to Europe $(\mathrm{BF}=494.52)$, Asia to North America $(\mathrm{BF}=30.94)$, Europe to Africa $(B F=21.47)$, South America to North America $(B F=19.99)$ (Figure 2a, Supplementary table 2). Markov rewards, which represent the mean proportion of time that viruses spend in each geographic region, demonstrated that IAVs circulate longest in Asia, followed by North America, and that continuous circulation in mainland Europe and Iceland is far less than the larger global regions (Supplementary Table 3a).

Continuous diffusion analyses further revealed the interconnectedness of Iceland isolates with lineages in mainland Europe and North America. Given the strong evolutionary and migratory connectivity between viruses isolated in Iceland with those isolated in Europe and North America, we sought to characterize the strength of association and directional flow of IAVs between intracontinental regions within and between mainland Europe, North America, and Iceland. Discrete phylogeographic diffusion modeling demonstrated with very strong statistical support $(\mathrm{BF}=110,622)$ that locations in mainland Europe (excluding Scandinavia) are the most significant sources of IAVs in Iceland (Markov jumps 5.37\%), followed by the northeastern region of the United States (Markov jumps 1.25\%). Iceland acted as a highly supported $(\mathrm{BF}=127.22)$ source region of IAVs to northeastern Canada and Greenland (Markov jumps 0.82\%) (Figure 3). Bayes factors and posterior probabilities for all intracontinental regional state transitions can be found in Supplementary Table 4 .

\section{Inter-species transmission dynamics}

Anseriformes and Charadriiformes were responsible for $59 \%$ and $41 \%$ of IAV introductions to Iceland, respectively (Supplementary figure 5). Local host-specific phylodynamic analyses within Iceland demonstrated that ducks $(\mathrm{BF}=6.05)$ and shorebirds $(\mathrm{BF}=5.57)$ drive transmission as sources of IAVs to gulls, while interspecies virus exchange occurs bi-directionally between shorebirds and ducks (shorebirds to ducks, $\mathrm{BF}=5.16$; ducks to shorebirds, $\mathrm{BF}=3.27$ ) (Figure 4a). Although geese in Iceland carry IAVs, there is no quantifiable inter-species transmission between geese and other species, which suggests a limited interface for geesederived transmission to occur in Iceland. The proportion of time viruses are hosted by a particular species, as derived from host-specific Markov rewards analyses, revealed that gulls host IAVs for the greatest proportion of time in Iceland (62.5\% versus $22.8 \%$ (geese), $8.4 \%$ (shorebirds), $6.3 \%$ (ducks)) (Supplementary Table $3 \mathrm{~b}$ ). This differs from global analyses where Anseriformes host IAVs for the greatest proportion of time as compared with other host orders (Supplementary Table 3c). Additionally, Anseriformes are the most significant drivers of transmission to other avian orders globally, including to Charadriiformes $(\mathrm{BF}=$ $29,353)$ and Galliformes $(\mathrm{BF}=29,353)$ (Supplementary figure 6). Median diffusion rates, a measure of how quickly hosts move viruses in space and time, were similar among shorebirds ( $577 \mathrm{~km} /$ year $(95 \% \mathrm{HPD}=$ $36.7-1844.2))$, ducks $(604 \mathrm{~km} /$ year $(95 \% \mathrm{HPD}=20.1-2768.6))$, gulls $(483 \mathrm{~km} /$ year $(95 \% \mathrm{HPD}=286-734.9))$, and geese $(386 \mathrm{~km} /$ year $(95 \% \mathrm{HPD}=194.6-640.9))$ within Iceland, with some extreme outliers among ducks and shorebirds (Figure $4 \mathrm{~b}$ ).

\section{Influenza subtype reassortment}

Reassortment primarily occurred among a limited number of subtypes, despite high subtype diversity in Iceland, including detection of 10 of $16 \mathrm{HA}$ and eight of nine NA avian subtypes over an eight-year period. Phylodynamic analyses of HA subtype reassortment within Iceland demonstrated significant reassortment among donor $\mathrm{H} 16$ subtypes to recipient $\mathrm{H} 2$ subtypes $(\mathrm{BF}=870.62)$, followed by $\mathrm{H} 3$ to $\mathrm{H} 10(\mathrm{BF}=21.35)$ and $\mathrm{H} 11(\mathrm{BF}=42.93)$, and $\mathrm{H} 2$ to $\mathrm{H} 10(\mathrm{BF}=19.08)$. Reassortment from donor $\mathrm{H} 3$ subtypes to recipient $\mathrm{H} 10$ and H11 subtypes was only detected in the Iceland-focused analysis and not detected in global analyses (Figure 5a and 5b). HA subtypes were largely host-specific, and HA reassortment patterns reflected inter-species transmission patterns described above (Figure 5c). IAV NA subtype reassortment in Iceland included N8 to $\mathrm{N} 7(\mathrm{BF}=6.97), \mathrm{N} 8$ to $\mathrm{N} 9(\mathrm{BF}=337.71), \mathrm{N} 5$ to $\mathrm{N} 7(\mathrm{BF}=3.47)$, and $\mathrm{N} 3$ to $\mathrm{N} 7(\mathrm{BF}=1358.31)$. Donor N5 subtypes to recipient N7 subtypes was the only reassortment pattern in Iceland that was not recapitulated in global analyses. H1 and N8 subtypes predominate across the global reassortment landscape as sources 
to almost all HA and NA subtypes (Figure 5). No low pathogenic to HPAI HA reassortment patterns were detected among Iceland isolates.

Discussion Our study is among the first to quantify the migratory connectivity of Iceland within the global context and the transmission dynamics that govern the spread of IAVs within Iceland and between adjacent geographic regions. Using Bayesian phylogeographic and phylodynamic inference, enabling the analysis of viral evolution simultaneously with ecological and population-level traits (Kuhnert, Wu, \& Drummond, 2011; Pybus \& Rambaut, 2009), we add insights into inter-hemispheric virus movement through, and inter-species transmission dynamics within, the North Atlantic subarctic, and Iceland specifically, a region that has largely been neglected in global phylogenetic analyses of IAVs (Hall et al., 2015; Herrick et al., 2013; The Global Consortium for H5N8 and Related Influenza Viruses, 2016).

Our findings reveal that $18 \%$ and five percent of global IAV transitions between regional states occur between a) mainland Europe and Iceland and b) North America and Iceland, respectively (Figure 2b). This demonstrates the importance of geographic proximity for inter-hemispheric movement of IAVs through the North Atlantic. This almost-fourfold difference is likely due to Iceland's geographical position along the East Atlantic flyway, which links western Africa and Europe and extends into Greenland and northeastern Canada via wild bird migration (Dusek et al., 2014). Additionally, previous research on avian migration in ASRs has suggested that birds avoid long-distance movements across the central Arctic Ocean and favor short distance flights along the great-circle orientation of the circumpolar north (Alerstam et al., 2007). Our findings further support this migration phenotype in the North Atlantic subarctic corridor, especially for the migratory transport of IAVs from mainland Europe to North America through intermediate staging locations in Iceland. Given the highly significant flow of IAVs from Asia to Europe, and mainland Europe to Iceland, many viruses are likely moving from Eurasia into Iceland along the east-west continuum into North America. This directional movement implies a higher risk than has been previously demonstrated for the flow of HPAI clade 2.3.4.4 viruses westward from Eurasia into North America.

Iceland serves as a highly transitional geography for migratory birds, converse to the North American system where birds tend to circulate for relatively longer periods of time (Supplementary table 3a). IAVs circulate much longer in North America (as compared with most other global regions aside from Asia) before transitioning to other global regions based on our Markov rewards analysis by global region (Supplementary table 3a). Long-term or endemic circulation in North America may be due to higher viral fitness relative to Eurasian origin clades (Bahl, Vijaykrishna, Holmes, Smith, \& Guan, 2009), or the geographic size of the continent, which may encompass the entire annual cycle for many species of birds (Hill et al., 2017). North Atlantic intraregional phylogeographic analyses demonstrate that northeastern Canada and Greenland are the most significant sink regions for IAVs following state transitions from Iceland (Figure 2). These data describe significant viral flow between Iceland and proximal regions in North America. Our findings reveal and quantify the inter-hemispheric movement of IAVs between mainland Europe and North America and the role that Iceland serves as an ecological 'land bridge' for wild birds and IAVs in space and time. The vast migratory range of gulls in the North Atlantic and their common routing through Iceland, suggests these avian hosts as particularly important for the inter-hemispheric transport of IAVs, though further research is necessary to describe the migratory connectivity of northern regions (Figure 3, Supplementary figure 7).

Iceland provides a staging ground for many migratory birds between mainland Europe and proximal regions, and therefore inter-species interactions during stopovers likely increase IAV transmission (Dusek et al., 2014). The importance of subarctic regions as sources of IAVs to southward latitudes was demonstrated through our discrete phylogeographic intracontinental analyses which not only determined Iceland as a strongly supported source to northeastern Canada, but also revealed a similarly strong relationship between Alaska and the western United States (Figure 1b, Supplementary Table 4). Alaska has been previously described as a significant stopover region for wild birds and thus a site for IAV incursion between Asia and North America (Hill et al., 2017; Wilson et al., 2013; Winker et al., 2007). Iceland and Alaska, therefore, serve as east-to-west and west-to-east incursion points for IAVs through northern latitudes into North America, respectively. Given that both Iceland and Alaska stopover locations serve as summer breeding grounds and inter-hemispheric 
bridges for long-distance migratory birds, increased surveillance in ASRs along migratory flyways is imperative to increase knowledge of IAV incursion into southward latitudes (Hill et al., 2017; Winker et al., 2007). Connections between Iceland and Alaska, however, based on our analyses are uncommon.

While Anseriformes drive transmission dynamics globally, host transmission phylodynamics in Iceland demonstrate the unique importance of gulls in this localized system. Gulls act as sinks and not sources of viruses to other host taxonomic groups in Iceland, and IAVs circulate within these seabirds for a far greater proportion of time than any of the other avian species we studied. Though Charadriiformes predominated in sequence count and hosted the highest genetic diversity of IAVs in both the original and downsampled datasets, our downsampling strategy reduced the number of sequence taxa for computation while mitigating sampling bias to maintain viral genetic diversity in the dataset. As such, IAV circulation time within gull populations may be due to the number of gulls in the dataset or host and virus ecological factors dictating continuous within-gull transmission patterns. Charadriiformes host a diversity of IAV subtypes, though two HA subtypes, H13 and H16, are primarily associated with Charadriiformes globally (Fouchier et al., 2005; Guinn et al., 2016; Hanson et al., 2008; Van Borm et al., 2012). In contrast, gulls in Iceland, hosted a wide range of IAV subtypes - several more than Anseriformes (Supplementary Table 1a). A similar diversity of IAV lineages and geographically reassorted viruses have been recorded in gulls at other high latitude locations (Huang et al., 2014; Wille et al., 2011), which may be due to the presence of large gull populations in ASRs, their pelagic long-distance migration between hemispheres (Figure 3), and opportunistic foraging strategies that characterize Charadriiformes generally (Callaghan, 2021; Van Borm et al., 2012; Wille et al., 2011; Wong, 2014). More research is necessary to describe the contribution of gulls to the global IAV reassortment landscape.

Our data demonstrate that gulls serve as recipient hosts of IAVs from ducks and shorebirds and there is limited onward transmission to other species, suggesting that gulls are permissive hosts but rarely amplify viruses from other hosts. Such transmission dynamics are likely guided by several species-specific behavioral and ecological factors. First, though many gull species inhabit oceanic coastlines, their scavenging behavior often takes them to diverse habitats, such as wetlands where ducks predominate, increasing opportunities for reservoir host duck to gull transmission of IAVs (Huang et al., 2014; Kubetzki \& Garthe, 2003). A large proportion of gulls staging in Iceland may be immuno-naive juveniles following the spring breeding season, which may explain the high susceptibility of gulls as recipients of duck-transmitted IAVs at these interfaces (Altizer, Bartel, \& Han, 2011; Hill \& Runstadler, 2016). Second, gulls can fly great distances over vast marine ecosystems and facilitate migratory connectivity between global regions (Dugan et al., 2008; Dusek et al., 2014; Van Borm et al., 2012). Many gull species transiently stage in Iceland before onward short- and long-distance migrations to adjacent locations in the region (Hallgrimsson, Gunnarsson, Torfason, Buijs, \& Camphuysen, 2012; Olsen KM, 2003; Wille et al., 2011), which may limit inter-species transmission opportunities from gulls to ducks and other avian taxa within Iceland.

Reassortment of IAV gene segments encoding HA surface glycoproteins is a mechanism for jumping between taxonomically distinct hosts (Ma, Hill, Zabilansky, Yuan, \& Runstadler, 2016) and has been implicated in the emergence of pandemic influenza viruses in humans (Ward, Lycett, Avila, Bollback, \& Leigh Brown, 2013). Though no reassortment between low pathogenic and HPAI HA subtypes was detected in Iceland during our eight-year sampling timeframe, we detected strong support for reassortment between gull-associated H16 to $\mathrm{H} 2$ in our sample (Figure 5). Both $\mathrm{H} 16$ and H2 subtypes were found at high prevalence among gulls in the study sample, demonstrating a reassortment pattern which may be due to the high frequency of both subtypes within this system and reflect a trend of sustained gull-to-gull transmission in Iceland. Though H2 subtype IAVs are diverse and distributed among two primary lineages (Zhuang et al., 2019), H2N2, the virus responsible for the 1957 pandemic, persists in wild migratory birds and commercial poultry in areas adjacent to the North Atlantic and other global regions (Glaser et al., 2006; Mo et al., 2021; Schafer et al., 1993; Shortridge, 1979). Historically, avian-origin H2 subtype IAVs have demonstrated spillover followed by sustained transmission among humans, therefore monitoring of $\mathrm{H} 2$ viruses in wild birds is considered important for evaluating spillover events into humans (Jones et al., 2014). We also detected significant levels of $\mathrm{H} 2$ and $\mathrm{H} 3$ to $\mathrm{H} 10$ subtype reassortment. $\mathrm{H} 10$ viruses have been frequently isolated from wild and domestic 
avian species in recent years, with the first human cases of H10N7 confirmed in Egypt in 2004 (El-Shesheny et al., 2018). An H10N7 subtype has also been associated with mass mortality events in harbor seals (Phoca vitulina ) throughout northern Europe (Bodewes et al., 2015). Active reassortment and circulation of H10 subtypes in the North Atlantic pose a risk to mammalian hosts, especially given native harbor seals inhabit temperate coastal regions in Iceland, southern Greenland, and northeastern North America (Teilmann \& Galatius, 2018).

We demonstrate novel significant findings describing IAV geographic movement, inter-species transmission, and reassortment in the North Atlantic region, although there are a few limitations to our analysis. First, despite eight consecutive years of sampling wild birds for IAVs in Iceland, the total number of fully and partially sequenced viruses to-date remain moderately low at 93 PB2 sequences from 92 individual birds. While our sampling effort was focused on those species groups most often associated with IAVs, sampling bias may have been introduced and other constraints may have impacted the detection, successful isolation, and sequencing of IAV gene segments. Potential sampling bias may have impacted findings related to the proportion of time IAVs spend within each host category. More systematic sampling across years may have provided an increased understanding of IAV dynamics in the North Atlantic region. Our downsampling strategy sought to limit the inclusion of such biases into our analyses. Second, while this analysis relied on one internal gene segment (PB2) to maximize the number of nucleotides in the analysis and investigate transmission dynamics without targeting a specific subtype, inclusion of multiple internal gene segments may have increased the robustness of and identified phylogenetic incongruence in the analysis, whereby different genes exhibit distinct phylogenies. Additionally, phylogenetic histories inferred from internal segments like PB2 may result in different conclusions to those from antigenic segments. Third, heterogenous sampling of IAVs across the globe and the computational limitations of discrete-trait Bayesian phylodynamics require that viruses from each region are downsampled to similarly sized groupings to avoid overrepresentation of certain traits (i.e., geographic region, host species, subtype) which can bias ancestral state reconstructions in the phylogenies. While we are confident our downsampling strategy evened groupings as much as possible while preserving viral, host, and temporal diversity across all regions, our approach does not remove the potential for sampling bias altogether. For example, we did not account for the geographic area nor population sizes of sampled regions, which could limit the inclusion of subpopulations and/or attenuate within-region migration dynamics (Kuhnert, Stadler, Vaughan, \& Drummond, 2016; Lemey et al., 2014).

Our findings reveal that IAV movement in arctic and subarctic regions follows wild bird migration around the perimeter of the circumpolar north which favors short-distance flights between proximal regions rather than long distance flights over the polar interior. Iceland connects mainland Europe and North America for the inter-hemispheric movement of IAVs, particularly due to the westward migration of wild birds from mainland Europe to Northeastern Canada and Greenland. We demonstrate that both Iceland and Alaska are incursion points of IAVs into North America and add evidence that northern regions in the subarctic are ecologically significant, where inter-continental mixing of disparate bird populations occurs and thus play an important role for inter-species transmission and onward dispersal of IAVs globally. Given that Iceland is situated along overlapping flyways that connect eastern and western hemispheres, its breeding and staging locations for migratory aquatic avian species are important sites for monitoring and surveillance of IAVs. Increased IAV surveillance in northern circumpolar regions may further reveal the source-sink dynamics between Eurasian and North American lineages. Further research could address ecologic and anthropogenic drivers of low and highly pathogenic IAV diffusion between hemispheres, and within the North Atlantic region specifically to support early warning for potential intercontinental incursion events into North America. As climate regimes in northern latitudes continue to shift, further monitoring of regional IAV dynamics may uncover important predictors of viral incursion of low and HPAI subtypes globally.

\section{Acknowledgements}

We acknowledge all contributions by hunters and fishers who provided hunter-harvested and fishery-bycatch sample collection over the eight-year timeframe of this project. Additionally, we would like to acknowledge field and laboratory staff who participated in this project over the years, including Herman Dreki Guls, Iris 
Myrdal Kristinsdottir, and Sindri Gislason for help with the capture and sampling of birds in Iceland, Sean Nashold and Joshua TeSlaa at the U.S. Geological Survey National Wildlife Health Center, and Tao Li, Wiriya Rutvisuttinunt, and Richard Jarman at the Walter Reed Army Institute of Research for sequencing virus isolates. We would like to acknowledge the Sudurnes Science and Learning Center, Sandgerdi, Iceland for providing lodging and laboratory support without which this project could not have succeeded. We also thank the reviewers for their comments and suggestions on previous versions of this article. The authors have no conflicts of interests to declare. This project was funded through the National Institute of Allergy and Infectious Diseases (grant \#s: HHSN272201400008C, HHSN272201400008C). Any use of trade, firm, or product names is for descriptive purposes only and does not imply endorsement by the U.S. Government. This article has been peer reviewed and approved for publication consistent with U.S. Geological Survey Fundamental Science Practices (https://pubs.usgs.gov/circ/1367/).

\section{References}

Alerstam, T., Backman, J., Gudmundsson, G. A., Hedenstrom, A., Henningsson, S. S., Karlsson, H., . . - Strandberg, R. (2007). A polar system of intercontinental bird migration. Proc Biol Sci, 274 (1625), 2523-2530. doi:10.1098/rspb.2007.0633

Altizer, S., Bartel, R., \& Han, B. A. (2011). Animal Migration and Infectious Disease Risk. Science, 331 (6015), 296-302. doi:10.1126/science.1194694

Antigua, K. J. C., Choi, W.-S., Baek, Y. H., \& Song, M.-S. (2019). The Emergence and Decennary Distribution of Clade 2.3.4.4 HPAI H5Nx.Microorganisms, 7 (6), 156. doi:10.3390/microorganisms7060156

Arctic Monitoring and Assessment Programme (AMAP). (2019). AMAP Climate Change Update 2019: An Update to Key Findings of Snow, Water, Ice and Permafrost in the Arctic . Retrieved from Oslo, Norway:

Baele, G., Ayres, D. L., Rambaut, A., Suchard, M. A., \& Lemey, P. (2019). High-Performance Computing in Bayesian Phylogenetics and Phylodynamics Using BEAGLE. In M. Anisimova (Ed.), Evolutionary Genomics: Statistical and Computational Methods (pp. 691-722). New York, NY: Springer New York.

Baele, G., Suchard, M. A., Rambaut, A., \& Lemey, P. (2017). Emerging Concepts of Data Integration in Pathogen Phylodynamics. Systematic Biology, 66 (1), e47-e65. doi:10.1093/sysbio/syw054

Bahl, J., Vijaykrishna, D., Holmes, E. C., Smith, G. J. D., \& Guan, Y. (2009). Gene flow and competitive exclusion of avian influenza A virus in natural reservoir hosts. Virology, 390 (2), 289-297. doi:10.1016/j.virol.2009.05.002

Bao, Y., Bolotov, P., Dernovoy, D., Kiryutin, B., Zaslavsky, L., Tatusova, T., . . . Lipman, D. (2008). The influenza virus resource at the National Center for Biotechnology Information. J Virol, 82 (2), 596-601. doi:10.1128/jvi.02005-07

Bodewes, R., Bestebroer, T. M., van der Vries, E., Verhagen, J. H., Herfst, S., Koopmans, M. P., . . . Osterhaus, A. D. M. E. (2015). Avian Influenza A(H10N7) virus-associated mass deaths among harbor seals.Emerging infectious diseases, 21 (4), 720-722. doi:10.3201/eid2104.141675

Butler, D. (2012). Flu surveillance lacking. Nature News, 483 (7391), 520.

Byrd-Leotis, L., Cummings, R. D., \& Steinhauer, D. A. (2017). The Interplay between the Host Receptor and Influenza Virus Hemagglutinin and Neuraminidase. International journal of molecular sciences, 18 (7), 1541. doi:10.3390/ijms18071541

Callaghan, C. (2021). Global abundance estimates for 9,700 bird species.Proc Natl Acad Sci U S A, 118 (21). doi:10.1073/pnas.2023170118

Causey, D., \& Edwards, S. V. (2008). Ecology of Avian Influenza Virus in Birds. The Journal of Infectious Diseases, 197 (Supplement_1), S29-S33. doi:10.1086/524991 
Chen, R., \& Holmes, E. C. (2006). Avian Influenza Virus Exhibits Rapid Evolutionary Dynamics. Molecular Biology and Evolution, 23 (12), 2336-2341. doi:10.1093/molbev/msl102

Chen, R., \& Holmes, E. C. (2009). Frequent inter-species transmission and geographic subdivision in avian influenza viruses from wild birds. Virology, 383 (1), 156-161. doi:10.1016/j.virol.2008.10.015

Dellicour, S., Rose, R., \& Pybus, O. G. (2016). Explaining the geographic spread of emerging epidemics: a framework for comparing viral phylogenies and environmental landscape data. BMC Bioinformatics, 17 (1), 82. doi:10.1186/s12859-016-0924-x

Docherty, D. E., \& Slota, P. G. (1988). Use of muscovy duck embryo fibroblasts for the isolation of viruses from wild birds. Journal of tissue culture methods, 11 (3), 165-170. doi:10.1007/BF01404270

Drummond, A. J., Ho, S. Y. W., Phillips, M. J., \& Rambaut, A. (2006). Relaxed Phylogenetics and Dating with Confidence. PLOS Biology, 4 (5), e88. doi:10.1371/journal.pbio.0040088

Dugan, V. G., Chen, R., Spiro, D. J., Sengamalay, N., Zaborsky, J., Ghedin, E., . . . Taubenberger, J. K. (2008). The Evolutionary Genetics and Emergence of Avian Influenza Viruses in Wild Birds. PLOS Pathogens, 4 (5), e1000076. doi:10.1371/journal.ppat.1000076

Dusek, R. J., Hall, J. S., Hallgrimsson, G. T., Vignisson, S. R., Ragnarsdottir, S. B., \& Jonsson, J. E. (2022). Surveillance for Avian Influenza Virus in Iceland, 2010 - 2018 [Dataset] . Retrieved from: https://doi.org/10.5066/P9ODQJML

Dusek, R. J., Hallgrimsson, G. T., Ip, H. S., Jonsson, J. E., Sreevatsan, S., Nashold, S. W., . . . Hall, J. S. (2014). North Atlantic Migratory Bird Flyways Provide Routes for Intercontinental Movement of Avian Influenza Viruses. PLOS ONE, 9 (3), e92075. doi:10.1371/journal.pone.0092075

El-Shesheny, R., Franks, J., Marathe, B. M., Hasan, M. K., Feeroz, M. M., Krauss, S., . . . Webster, R. G. (2018). Genetic characterization and pathogenic potential of H10 avian influenza viruses isolated from live poultry markets in Bangladesh. Scientific Reports, 8 (1), 10693-10693. doi:10.1038/s41598-018-29079-1

Fouchier, R. A., Munster, V., Wallensten, A., Bestebroer, T. M., Herfst, S., Smith, D., . . . Osterhaus, A. D. (2005). Characterization of a novel influenza A virus hemagglutinin subtype (H16) obtained from black-headed gulls. J Virol, 79 (5), 2814-2822. doi:10.1128/jvi.79.5.2814-2822.2005

Gass J.D. (2021). Global dissemination of Influenza A virus is driven by wild bird migration through arctic and subarctic zones [Dataset]. Dryad Digital Repository. https://doi.org/10.5061/dryad.m37pvmd2m .

Gilbert, M., Slingenbergh, J., \& Xiao, X. (2008). Climate change and avian influenza. Revue scientifique et technique (International Office of Epizootics), 27 (2), 459-466.

Glaser, L., Zamarin, D., Acland, H. M., Spackman, E., Palese, P., Garcia-Sastre, A., \& Tewari, D. (2006). Sequence analysis and receptor specificity of the hemagglutinin of a recent influenza H2N2 virus isolated from chicken in North America. Glycoconjugate Journal, 23 (1), 93-99. doi:10.1007/s10719-006-5441-0

Guan, M., Hall, J. S., Zhang, X., Dusek, R. J., Olivier, A. K., Liu, L., . . . Wan, X.-F. (2019). Aerosol Transmission of Gull-Origin Iceland Subtype H10N7 Influenza A Virus in Ferrets. Journal of Virology, 93 (13), e00282-00219. doi:10.1128/jvi.00282-19

Gudmundsson, G. A. (1993). The spring migration pattern of arctic birds in southwest Iceland, as recorded by radar. Ibis, 135 (2), 166-176. doi:10.1111/j.1474-919X.1993.tb02828.x

Gudmundsson, L. A. (1992). Spring migration of sanderlings Calidris alba through SW Iceland: Wherefrom and whereto? ARDEA, 80, 315-326.

Guinn, K., Fojtik, A., Davis-Fields, N., Poulson, R. L., Krauss, S., Webster, R. G., \& Stallknecht, D. E. (2016). Antibodies to Influenza A Viruses in Gulls at Delaware Bay, USA. Avian diseases, 60 (1s), 341-345. doi:10.1637/11103-042115-Reg 
Hall, J. S., Hallgrimsson, G. T., Suwannanarn, K., Sreevatsen, S., Ip, H. S., Magnusdottir, E., . . . Dusek, R. J. (2014). Avian influenza virus ecology in Iceland shorebirds: Intercontinental reassortment and movement. Infection, Genetics and Evolution, 28 , 130-136. doi:https://doi.org/10.1016/j.meegid.2014.09.013

Hall, J. S., Russell, R. E., Franson, J. C., Soos, C., Dusek, R. J., Allen, R. B., . . . Brown, J. D. (2015). Avian Influenza Ecology in North Atlantic Sea Ducks: Not All Ducks Are Created Equal. PLOS ONE, 10 (12), e0144524. doi:10.1371/journal.pone.0144524

Hall, J. S., TeSlaa, J. L., Nashold, S. W., Halpin, R. A., Stockwell, T., Wentworth, D. E., . . . Ip, H. S. (2013). Evolution of a reassortant North American gull influenza virus lineage: drift, shift and stability. Virology Journal, 10 (1), 179. doi:10.1186/1743-422x-10-179

Hallgrimsson, G. T., Gunnarsson, H. V., Torfason, O., Buijs, R.-J., \& Camphuysen, K. C. J. (2012). Migration pattern of Icelandic Lesser Black-backed Gulls Larus fuscus graellsii: indications of a leap-frog system. Journal of Ornithology, 153 (3), 603-609. doi:10.1007/s10336-012-0816-4

Hanson, B. A., Luttrell, M. P., Goekjian, V. H., Niles, L., Swayne, D. E., Senne, D. A., \& Stallknecht, D. E. (2008). IS THE OCCURRENCE OF AVIAN INFLUENZA VIRUS IN CHARADRIIFORMES SPECIES AND LOCATION DEPENDENT? Journal of Wildlife Diseases, 44 (2), 351-361. doi:10.7589/0090-355844.2.351

Henaux, V., Parmley, J., Soos, C., \& Samuel, M. D. (2013). Estimating transmission of avian influenza in wild birds from incomplete epizootic data: implications for surveillance and disease spread. Journal of Applied Ecology, 50 (1), 223-231. doi:10.1111/1365-2664.12031

Herrick, K. A., Huettmann, F., \& Lindgren, M. A. (2013). A global model of avian influenza prediction in wild birds: the importance of northern regions. Veterinary Research, 44 (1), 42. doi:10.1186/1297-9716-4442

Hicks, J. T., Dimitrov, K. M., Afonso, C. L., Ramey, A. M., \& Bahl, J. (2019). Global phylodynamic analysis of avian paramyxovirus-1 provides evidence of inter-host transmission and intercontinental spatial diffusion. BMC evolutionary biology, 19 (1), 108. doi:10.1186/s12862-019-1431-2

Hill, N., Hussein, I., Davis, K., Ma, E., Spivey, T., Ramey, A., . . . Runstadler, J. (2017). Reassortment of Influenza A Viruses in Wild Birds in Alaska before H5 Clade 2.3.4.4 Outbreaks. Emerging infectious diseases, 23 (4), 654-657. doi:10.3201/eid2304.161668

Hill, N., Ma, E., Meixell, B., Lindberg, M., Boyce, W., \& Runstadler, J. (2016). Transmission of influenza reflects seasonality of wild birds across the annual cycle. Ecology Letters, 19 (8), 915-925. doi:10.1111/ele.12629

Hill, N., \& Runstadler, J. (2016). A Bird's Eye View of Influenza A Virus Transmission: Challenges with Characterizing Both Sides of a Co-Evolutionary Dynamic. Integr Comp Biol, 56 (2), 304-316. doi:10.1093/icb/icw055

Hong, S. L., Lemey, P., Suchard, M. A., \& Baele, G. (2021). Bayesian Phylogeographic Analysis Incorporating Predictors and Individual Travel Histories in BEAST. Current Protocols, 1 (4), e98. doi:https://doi.org/10.1002/cpz1.98

Hoye, B. J., Munster, V. J., Nishiura, H., Fouchier, R. A. M., Madsen, J., \& Klaassen, M. (2011). Reconstructing an annual cycle of interaction: natural infection and antibody dynamics to avian influenza along a migratory flyway. Oikos, 120 (5), 748-755. doi:https://doi.org/10.1111/j.1600-0706.2010.18961.x

Hoye, B. J., Munster, V. J., Nishiura, H., Klaassen, M., \& Fouchier, R. A. M. (2010). Surveillance of wild birds for avian influenza virus.Emerging infectious diseases, 16 (12), 1827-1834. doi:10.3201/eid1612.100589

Huang, Y., Wille, M., Benkaroun, J., Munro, H., Bond, A. L., Fifield, D. A., . . . Lang, A. S. (2014). Perpetuation and reassortment of gull influenza A viruses in Atlantic North America. Virology, 456-457, 353-363. doi:https://doi.org/10.1016/j.virol.2014.04.009 
Ito, T., Okazaki, K., Kawaoka, Y., Takada, A., Webster, R. G., \& Kida, H. (1995). Perpetuation of influenza A viruses in Alaskan waterfowl reservoirs. Arch Virol, 140 (7), 1163-1172. doi:10.1007/bf01322743

IUCN. (2021). The IUCN Red List of Threatened Species. Version 2021-3. https://www.iucnredlist.org. Accessed on 07/03/2021.

Jensen, R., Madsen, J., O'Connell, M., Wisz, M., Tommervik, S., \& F, M. (2008). Prediction of the distribution of Arctic-nesting pink-footed geese under a warmer climate scenario. Global Change Biology, 14 (1), 1-10. doi:https://doi.org/10.1111/j.1365-2486.2007.01461.x

JMP Version 14.0.0. (1989-2019): SAS Institute Inc., Cary, NC.

Jones, J. C., Baranovich, T., Marathe, B. M., Danner, A. F., Seiler, J. P., Franks, J., . . . Webster, R. G. (2014). Risk assessment of H2N2 influenza viruses from the avian reservoir. Journal of Virology, 88 (2), 1175-1188. doi:10.1128/JVI.02526-13

Kass, R. E., \& Raftery, A. E. (1995). Bayes Factors. Journal of the American Statistical Association, 90 (430), 773-795. doi:10.1080/01621459.1995.10476572

Kilpatrick, A. M., Chmura, A. A., Gibbons, D. W., Fleischer, R. C., Marra, P. P., \& Daszak, P. (2006). Predicting the global spread of H5N1 avian influenza. Proceedings of the National Academy of Sciences, 103 (51), 19368-19373. doi:10.1073/pnas.0609227103

Kubetzki, U., \& Garthe, S. (2003). Distribution, diet and habitat selection by four sympatrically breeding gull species in the south-eastern North Sea. Marine Biology, 143 (1), 199-207. doi:10.1007/s00227-003-10365

Kuhnert, D., Stadler, T., Vaughan, T. G., \& Drummond, A. J. (2016). Phylodynamics with Migration: A Computational Framework to Quantify Population Structure from Genomic Data. Molecular Biology and Evolution, 33 (8), 2102-2116. doi:10.1093/molbev/msw064

Kuhnert, D., Wu, C.-H., \& Drummond, A. J. (2011). Phylogenetic and epidemic modeling of rapidly evolving infectious diseases.Infection, Genetics and Evolution, 11 (8), 1825-1841. doi:https://doi.org/10.1016/j.meegid.2011.08.005

Lang, A. S., Lebarbenchon, C., Ramey, A. M., Robertson, G. J., Waldenstrom, J., \& Wille, M. (2016). Assessing the Role of Seabirds in the Ecology of Influenza A Viruses. Avian diseases, 60 (1s), 378-386. doi:10.1637/11135-050815-RegR

Lee, D. H., Bahl, J., Torchetti, M. K., Killian, M. L., Ip, H. S., DeLiberto, T. J., \& Swayne, D. E. (2016). Highly Pathogenic Avian Influenza Viruses and Generation of Novel Reassortants, United States, 2014-2015. Emerg Infect Dis, 22 (7), 1283-1285. doi:10.3201/eid2207.160048

Lee, D. H., Bertran, K., Kwon, J. H., \& Swayne, D. E. (2017). Evolution, global spread, and pathogenicity of highly pathogenic avian influenza H5Nx clade 2.3.4.4. J Vet Sci, 18 (S1), 269-280. doi:10.4142/jvs.2017.18.S1.269

Lee, D. H., Torchetti, M. K., Winker, K., Ip, H. S., Song, C. S., \& Swayne, D. E. (2015). Intercontinental Spread of Asian-Origin H5N8 to North America through Beringia by Migratory Birds. J Virol, 89 (12), 6521-6524. doi:10.1128/jvi.00728-15

Lemey, P., Rambaut, A., Bedford, T., Faria, N., Bielejec, F., Baele, G., . . . Suchard, M. A. (2014). Unifying Viral Genetics and Human Transportation Data to Predict the Global Transmission Dynamics of Human Influenza H3N2. PLOS Pathogens, 10 (2), e1003932. doi:10.1371/journal.ppat.1003932

Lemey, P., Rambaut, A., Drummond, A. J., \& Suchard, M. A. (2009). Bayesian Phylogeography Finds Its Roots. PLoS computational biology, 5 (9), e1000520. doi:10.1371/journal.pcbi.1000520 
Lemey, P., Rambaut, A., Welch, J. J., \& Suchard, M. A. (2010). Phylogeography takes a relaxed random walk in continuous space and time.Molecular Biology and Evolution, 27 (8), 1877-1885. doi: $10.1093 / \mathrm{molbev} / \mathrm{msq} 067$

Long, J. S., Mistry, B., Haslam, S. M., \& Barclay, W. S. (2019). Host and viral determinants of influenza A virus species specificity.Nature Reviews Microbiology, 17 (2), 67-81. doi:10.1038/s41579-018-0115-Z

Ma, E. J., Hill, N. J., Zabilansky, J., Yuan, K., \& Runstadler, J. A. (2016). Reticulate evolution is favored in influenza niche switching.Proceedings of the National Academy of Sciences of the United States of America, 113 (19), 5335-5339.

Margulies, M., Egholm, M., Altman, W. E., Attiya, S., Bader, J. S., Bemben, L. A., . . . Rothberg, J. M. (2005). Genome sequencing in microfabricated high-density picolitre reactors. Nature, 437 (7057), 376-380. doi:10.1038/nature03959

Mckinney, M. A., Pedro, S., Dietz, R., Sonne, C., Fisk, A. T., Roy, D., . . . Letcher, R. J. (2015). A review of ecological impacts of global climate change on persistent organic pollutant and mercury pathways and exposures in arctic marine ecosystems. Current Zoology, 61 (4), 617-628. doi:10.1093/czoolo/61.4.617

Minin, V. N., Bloomquist, E. W., \& Suchard, M. A. (2008). Smooth skyride through a rough skyline: Bayesian coalescent-based inference of population dynamics. Molecular Biology and Evolution, 25 (7), 14591471. doi:10.1093/molbev/msn090

Minin, V. N., \& Suchard, M. A. (2008). Counting labeled transitions in continuous-time Markov models of evolution. Journal of Mathematical Biology, 56 (3), 391-412. doi:10.1007/s00285-007-0120-8

Mo, J., Youk, S., Pantin-Jackwood, M. J., Suarez, D. L., Lee, D.-H., Killian, M. L., . . . Spackman, E. (2021). The pathogenicity and transmission of live bird market H2N2 avian influenza viruses in chickens, Pekin ducks, and guinea fowl. Vet Microbiol, 260 , 109180. doi:https://doi.org/10.1016/j.vetmic.2021.109180

Newton, I. (2008). The Migration Ecology of Birds : Academic Press.

Olsen, B., Munster, V. J., Wallensten, A., Waldenstrom, J., Osterhaus, A. D. M. E., \& Fouchier, R. A. M. (2006). Global Patterns of Influenza A Virus in Wild Birds. Science, 312 (5772), 384-388. doi:10.1126/science.1122438

Olsen KM, L. H. (2003). Gulls of Europe, Asia, and North America . London: A\&C Black Publishers Ltd.

Olsen KM, L. H. (2004). Gulls of Europe, Asia and North America(2nd Edition ed.). Princeton, New Jersey: Princeton University Press.

Olson, S. H., Parmley, J., Soos, C., Gilbert, M., Latorre-Margalef, N., Hall, J. S., . . . Joly, D. (2014). Sampling Strategies and Biodiversity of Influenza A Subtypes in Wild Birds. PLOS ONE, 9 (3), e90826. doi:10.1371/journal.pone.0090826

Pagel, M., Meade, A., \& Barker, D. (2004). Bayesian Estimation of Ancestral Character States on Phylogenies. Systematic Biology, 53 (5), 673-684. doi:10.1080/10635150490522232

Pearce, J. M., Ramey, A. M., Flint, P. L., Koehler, A. V., Fleskes, J. P., Franson, J. C., . . . Ip, H. S. (2009). Avian influenza at both ends of a migratory flyway: characterizing viral genomic diversity to optimize surveillance plans for North America. Evolutionary applications, 2 (4), 457-468. doi:https://doi.org/10.1111/j.1752-4571.2009.00071.x

Prater, A., Marchant, J., \& Vuorinen, J. (1977). Guide to the identification and ageing of Holarctic waders: BTO Guide No. 17 : BTO, Tring.

Pybus, O. G., \& Rambaut, A. (2009). Evolutionary analysis of the dynamics of viral infectious disease. Nature Reviews Genetics, 10 , 540. doi:10.1038/nrg2583 
QGIS Development Team. (2020). QGIS Geographic Information System. Retrieved from http://qgis.osgeo.org

Rambaut, A., Drummond, A. J., Xie, D., Baele, G., \& Suchard, M. A. (2018). Posterior Summarization in Bayesian Phylogenetics Using Tracer 1.7. Systematic Biology, 67 (5), 901-904. doi:10.1093/sysbio/syy032

Rambaut, A., Lam, T. T., Max Carvalho, L., \& Pybus, O. G. (2016). Exploring the temporal structure of heterochronous sequences using TempEst (formerly Path-O-Gen). Virus evolution, 2 (1), vew007-vew007. doi:10.1093/ve/vew007

Rambaut A. (2018). FigTree v 1.4. 4. In.

Ramey, Hill NJ, Deliberto TJ, Gibbs S, Hopkins MC, Lang AS, . . . XF, W. (2021). Highly pathogenic avian influenza is an emerging disease threat to North American wildlife [in press]. Journal of Wildlife Management .

Ramey, A., Reeves, A., Sonsthagen, S., TeSlaa, J., Nashold, S., Donnelly, T., . . . Hall, J. (2015). Dispersal of H9N2 influenza A viruses between East Asia and North America by wild birds.Virology, 482 , 79-83. doi:https://doi.org/10.1016/j.virol.2015.03.028

Ramey, A., Reeves, A., TeSlaa, J., Nashold, S., Donnelly, T., Bahl, J., \& Hall, J. (2016). Evidence for common ancestry among viruses isolated from wild birds in Beringia and highly pathogenic intercontinental reassortant $\mathrm{H} 5 \mathrm{~N} 1$ and $\mathrm{H} 5 \mathrm{~N} 2$ influenza A viruses. Infection, genetics and evolution : journal of molecular epidemiology and evolutionary genetics in infectious diseases, 40 , 176-185. doi:10.1016/j.meegid.2016.02.035

Ramey, A. M., Reeves, A. B., Drexler, J. Z., Ackerman, J. T., De La Cruz, S., Lang, A. S., . . . Stallknecht, D. E. (2020). Influenza A viruses remain infectious for more than seven months in northern wetlands of North America. Proceedings of the Royal Society B: Biological Sciences, 287 (1934), 20201680. doi:doi:10.1098/rspb.2020.1680

Runstadler, J., Hill, N., Hussein, I. T. M., Puryear, W., \& Keogh, M. (2013). Connecting the study of wild influenza with the potential for pandemic disease. Infection, genetics and evolution : journal of molecular epidemiology and evolutionary genetics in infectious diseases, 17 , 162-187. doi:10.1016/j.meegid.2013.02.020

Sanger, F., Nicklen, S., \& Coulson, A. R. (1977). DNA sequencing with chain-terminating inhibitors. Proceedings of the National Academy of Sciences of the United States of America, 74 (12), 5463-5467. doi:10.1073/pnas.74.12.5463

Schafer, J. R., Kawaoka, Y., Bean, W. J., Suss, J., Senne, D., \& Webster, R. G. (1993). Origin of the pandemic $1957 \mathrm{H} 2$ influenza A virus and the persistence of its possible progenitors in the avian reservoir. Virology, 194 (2), 781-788. doi:10.1006/viro.1993.1319

Shortridge, K. F. (1979). H2N2 influenza viruses in domestic ducks.Lancet, 1 (8113), 439. doi:10.1016/s01406736(79)90911-5

Spackman, E., Senne, D. A., Myers, T. J., Bulaga, L. L., Garber, L. P., Perdue, M. L., . . . Suarez, D. L. (2002). Development of a real-time reverse transcriptase PCR assay for type A influenza virus and the avian H5 and H7 hemagglutinin subtypes. Journal of Clinical Microbiology, 40 (9), 3256-3260. doi:10.1128/jcm.40.9.3256-3260.2002

Stamatakis, A. (2006). RAxML-VI-HPC: maximum likelihood-based phylogenetic analyses with thousands of taxa and mixed models.Bioinformatics, 22 (21), 2688-2690. doi:10.1093/bioinformatics/btl446

Suchard, M. A., Lemey, P., Baele, G., Ayres, D. L., Drummond, A. J., \& Rambaut, A. (2018). Bayesian phylogenetic and phylodynamic data integration using BEAST 1.10. Virus evolution, 4 (1). doi:10.1093/ve/vey016

Tavare, S., \& Miura, R. M. (1986). Lectures on mathematics in the life sciences. Paper presented at the Am. Math. Soc. 
Teilmann, J., \& Galatius, A. (2018). Harbor Seal: Phoca vitulina. In B. Wursig, J. G. M. Thewissen, \& K. M. Kovacs (Eds.), Encyclopedia of Marine Mammals (Third Edition) (pp. 451-455): Academic Press.

The Global Consortium for H5N8 and Related Influenza Viruses. (2016). Role for migratory wild birds in the global spread of avian influenza H5N8. Science, 354 (6309), 213-217. doi:10.1126/science.aaf8852

Trovao, N. S., Suchard, M. A., Baele, G., Gilbert, M., \& Lemey, P. (2015). Bayesian Inference Reveals HostSpecific Contributions to the Epidemic Expansion of Influenza A H5N1. Molecular Biology and Evolution, 32 (12), 3264-3275. doi:10.1093/molbev/msv185

USGS. (2020). Age (Year Class) Codes. Retrieved from https://www.pwrc.usgs.gov/bbl/manual/age.cfm

Van Borm, S., Rosseel, T., Vangeluwe, D., Vandenbussche, F., van den Berg, T., \& Lambrecht, B. (2012). Phylogeographic analysis of avian influenza viruses isolated from Charadriiformes in Belgium confirms intercontinental reassortment in gulls. Archives of Virology, 157 (8), 1509-1522. doi:10.1007/s00705-012-1323-x

van Dijk, J. G. B., Hoye, B. J., Verhagen, J. H., Nolet, B. A., Fouchier, R. A. M., \& Klaassen, M. (2014). Juveniles and migrants as drivers for seasonal epizootics of avian influenza virus. Journal of Animal Ecology, 83 (1), 266-275. doi:10.1111/1365-2656.12131

Verhagen, J. H., Hofle, U., van Amerongen, G., van de Bildt, M., Majoor, F., Fouchier, R. A. M., \& Kuiken, T. (2015). Long-Term Effect of Serial Infections with H13 and H16 Low-Pathogenic Avian Influenza Viruses in Black-Headed Gulls. Journal of Virology, 89 (22), 11507-11522. doi:10.1128/JVI.01765-15

Ward, M. J., Lycett, S. J., Avila, D., Bollback, J. P., \& Leigh Brown, A. J. (2013). Evolutionary interactions between haemagglutinin and neuraminidase in avian influenza. BMC evolutionary biology, 13 (1), 222. doi:10.1186/1471-2148-13-222

Webster, M. S., Marra, P. P., Haig, S. M., Bensch, S., \& Holmes, R. T. (2002). Links between worlds: unraveling migratory connectivity. Trends in Ecology $\&$ Evolution, 17 (2), 76-83. doi:https://doi.org/10.1016/S01695347(01)02380-1

Wille, M., Robertson, G. J., Whitney, H., Bishop, M. A., Runstadler, J. A., \& Lang, A. S. (2011). Extensive Geographic Mosaicism in Avian Influenza Viruses from Gulls in the Northern Hemisphere. PLOS ONE, 6 (6), e20664. doi:10.1371/journal.pone.0020664

Wilson, H. M., Hall, J. S., Flint, P. L., Franson, J. C., Ely, C. R., Schmutz, J. A., \& Samuel, M. D. (2013). High Seroprevalence of Antibodies to Avian Influenza Viruses among Wild Waterfowl in Alaska: Implications for Surveillance. PLOS ONE, 8 (3), e58308. doi:10.1371/journal.pone.0058308

Winker, K., McCracken, K. G., Gibson, D. D., Pruett, C. L., Meier, R., Huettmann, F., . . . Swayne, D. E. (2007). Movements of birds and avian influenza from Asia into Alaska. Emerging infectious diseases, 13 (4), 547-552. doi:10.3201/eid1304.061072

Wong, S. N. P. (2014). Hotspots in cold seas: The composition, distribution, and abundance of marine birds in the North American Arctic. Journal of Geophysical Research: Oceans, 119 (3), 1691-1705. doi:https://doi.org/10.1002/2013JC009198

Woolcock, P. R. (2008). Avian influenza virus isolation and propagation in chicken eggs. Methods in molecular biology (Clifton, N.J.), 436 , 35-46. doi:10.1007/978-1-59745-279-3_6

Yang, Z. (1996). Maximum-likelihood models for combined analyses of multiple sequence data. Journal of Molecular Evolution, 42 (5), 587-596. doi:10.1007/BF02352289

Zhuang, Q., Wang, S., Liu, S., Hou, G., Li, J., Jiang, W., . . . Chen, J. (2019). Diversity and distribution of type A influenza viruses: an updated panorama analysis based on protein sequences. Virology Journal, 16 (1), 85. doi:10.1186/s12985-019-1188-7

\section{Data Accessibility}


All data supporting the findings of this study are openly accessible. Genetic sequences from IAV surveillance in Iceland are deposited in GenBank (https://www.ncbi.nlm.nih.gov/genbank) under accession numbers listed in Supplementary table 1b. Raw data used for the analysis are deposited in the Dryad Digital Repository (https://doi.org/10.5061/dryad.m37pvmd2m).

\section{Author Contributions}

JDG designed and conducted all analyses and was the main writer of the paper. RJD, JSH, GTH, JDG, and NJH conceived of the study. RJD, GTH, HPH, SRV, SBR and JEJ directed and performed field work. JSH directed and performed laboratory assays of field samples. SK, SSW, XFW, SA, and SS directed and performed genetic sequencing of avian influenza isolates. NST provided mentorship for BEAST xml coding and Bayesian phylodynamic methods. FBN and JAR provided advisory mentorship to JDG. NJH provided senior mentorship to JDG including Bayesian phylodynamic methods and BEAST coding. All co-authors read the manuscript and provided edits and feedback during the writing process.

\section{Figures}

Figure 1. Markov Chain Monte Carlo (MCC) time-scaled phylogeographic tree and phylogeographic map of global downsampled Influenza A virus (IAV) sequences. (1.a) Phylogeographic tree, color-coded by geographic source region. Grey branches indicate historical sequences between 1978 and 2008 which contributed to the overall tree structure but were masked from phylogeographic analyses (Gass J.D., 2021). (1.b) phylogeographic map of global downsampled IAV sequences, color-coded by geographic source region. 95\% HPD tree and posterior value support can be found in Supplementary figure 4.

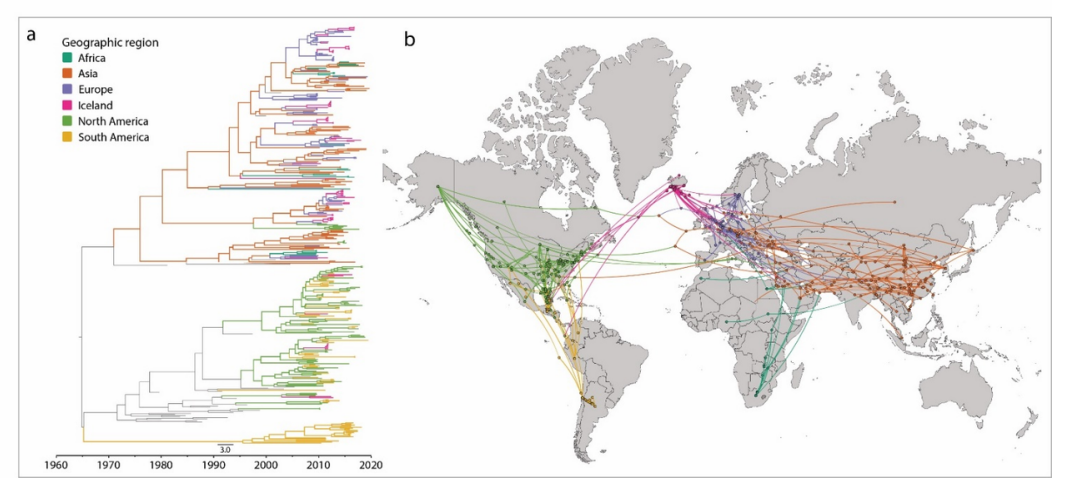

Figure 2. Global virus transitions and movement between regions. 2a) Discrete phylogeographic transitions between regional states. Transitions represent virus movement between regional states, measured by Bayes factor support (S.A.: South America, N.A.: North America). Cells with Bayes factor values above 3.0 are framed by a green border (Kass \& Raftery, 1995). 2b) Markov jumps, which are represented as the percent of global transitions between regional states along phylogenetic tree branches. Global transitions $<1 \%$ are not shown. 

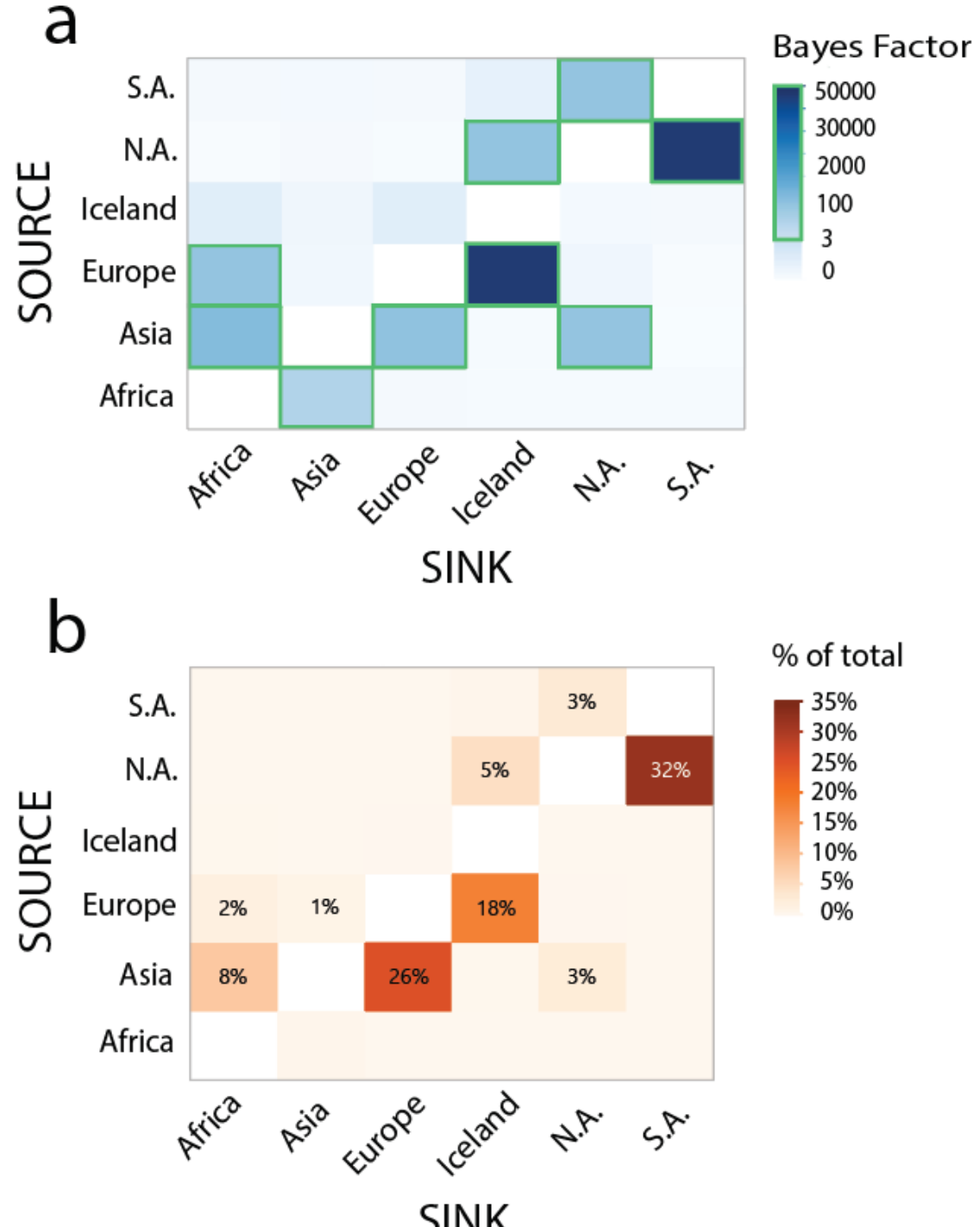

$\%$ of total

$35 \%$

$30 \%$

$25 \%$

$20 \%$

$15 \%$

$10 \%$

$5 \%$

$0 \%$

Figure 3. Host migratory distribution and IAV circulation in the North Atlantic. Significant discrete phylogeographic transitions between Iceland, the rest of Europe, and North America are represented by arrows from mainland Europe to Iceland (Bayes Factor $(B F)=110,622)$, Northeastern USA to Iceland $(B F=$ 414.87), and Iceland to northeastern Canada and Greenland $(\mathrm{BF}=127.22)$. Arrows signify directionality and greater arrow width corresponds to higher BF support of phylogeographic transitions between intracontinental geographic states. BFs for state transitions between all global regions can be found in Supplementary Table 4. Host migratory ranges for populations of shorebirds (yellow), gulls (green), ducks (blue), and geese (red) were compiled from individual species-specific shape files acquired from IUCN Red List (IUCN, 2021). Species-specific migratory range maps can be found in the Supplementary figure 7. 


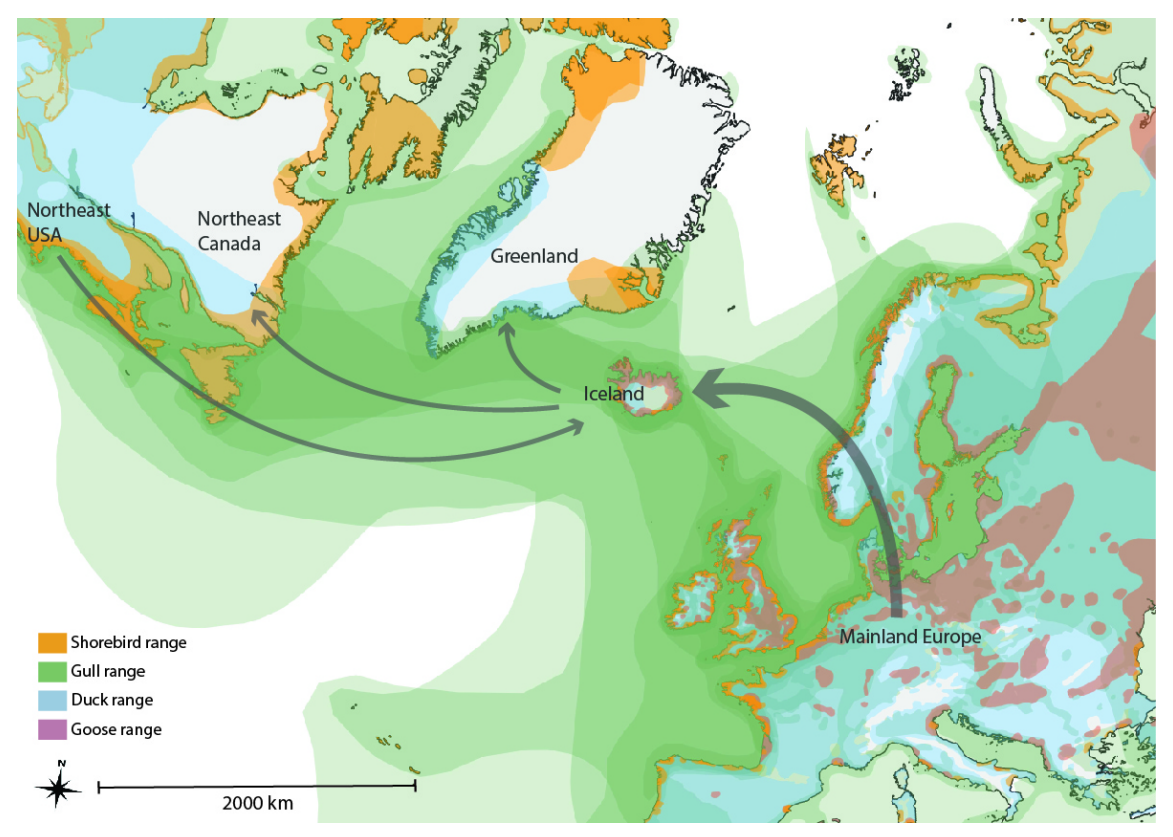

Figure 4. Discrete and continuous host-specific transmission dynamics and virus movement in Iceland. a) Transitions and Bayes Factors of IAV transmission between host taxa, and b) diffusion rates (km/year), by host taxa.
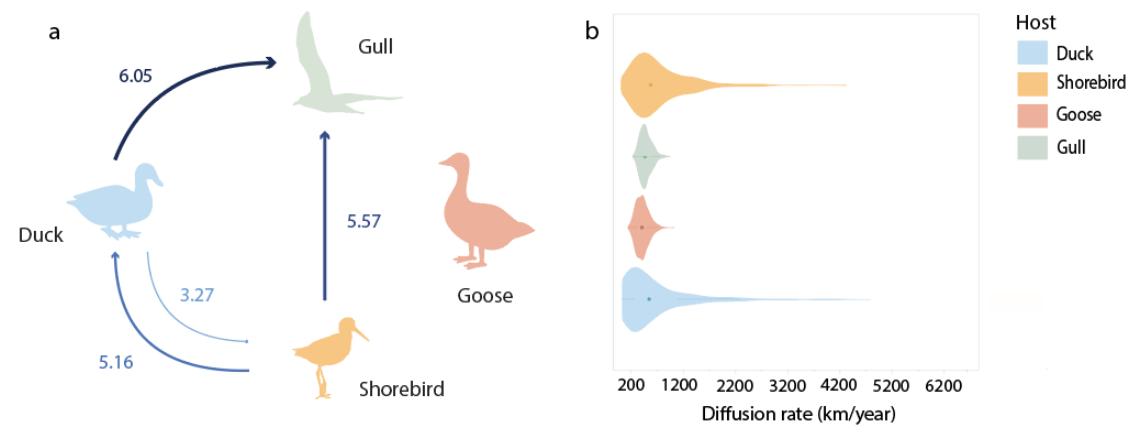

Figure 5. Global and Iceland-specific reassortment patterns and Bayes factors among Hemagglutinin (HA) subtypes (5a and b), Multiple Correspondence Analysis of Host order and HA subtype (5c), and reassortment patterns and Bayes factors among Neuraminidase (NA) subtypes (5d and e). a) The global reassortment matrix of HA subtypes demonstrates the breadth and highly significant transitions between donor H1 subtypes and almost all other recipient subtypes. b) In Iceland, a strong association between donor H16 subtypes and recipient $\mathrm{H} 2$ subtypes were found, as well as other significant transitions between $\mathrm{H} 3$ and $\mathrm{H} 10$ and H11 subtypes and $\mathrm{H} 2$ and $\mathrm{H} 10$ subtypes. Both forward and reverse rates are visualized in these matrices, with subtypes labeled on the $\mathrm{x}$-axis representing recipients and subtypes labeled on the $\mathrm{y}$-axis representing donors. Bayes factors, signifying the level of significance, are represented as colors from beige (low) to dark red (high). c) Multiple correspondence analysis (MCA) of HA subtype by is presented host order. Size of blue rectangles for HA subtype corresponds to prevalence in the dataset. d) Global and e) Iceland-specific reassortment patterns and Bayes factors (BF) among Neuraminidase (NA) subtypes. The global reassortment matrix demonstrates the breadth and highly significant transitions between donor N8 subtypes and almost all other recipient subtypes. In Iceland, a strong association between donor N3 subtypes and recipient 
N7 subtypes were found, as well as other significant transitions between N8 and N9 and N7 subtypes. Both forward and reverse rates are visualized in these matrices, with subtypes labeled on the $\mathrm{x}$-axis representing recipients and subtypes labeled on the y-axis representing donors. Bayes factors, signifying the level of significance, are represented as colors from white (low) to dark green (high).
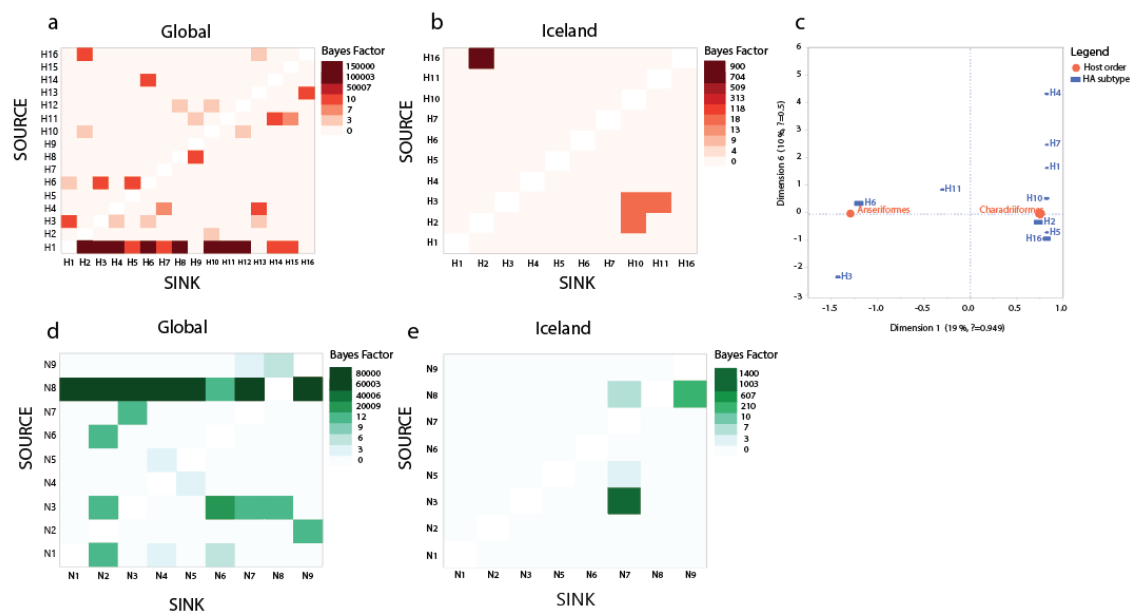

\section{Supporting information}

Supplemental information Iceland phylodynamics 20210811.doc 